\title{
Erratum to 10.1007/s11577-015-0303-z
}

\section{Sozialpsychologie}

Im Jahresinhaltverzeichnis des Jahres 2014 wurden leider einige Seitenzahlen nicht korrekt angegeben. Im Folgenden finden Sie die korrigierte Version des Verzeichnisses:

\section{LISTE DER AUTORINNEN UND AUTOREN DES 66. JAHRGANGS 2014 1*}

$\begin{array}{llll}\text { Akyel, Dominic } & 425 & \text { Lois, Daniel } & 417^{*} \\ \text { Allerbeck, Klaus } & 137 & \text { Lomi, Alessandro } & 395^{*} \\ \text { Andreß, Hans-Jürgen } & 575 & \text { Mayerl, Jochen } & 135^{*} \\ \text { Arránz Becker, Oliver } & 417^{*} & \text { Maynt, Renate } & 1 \\ \text { Auspurg, Katrin } & 21,549 & \text { Mentjox, Tessel } & 241^{*} \\ \text { Becker, Birgit } & 219 & \text { Menzl, Marcus } & 494 \\ \text { Beckert, Jens } & 425 & \text { Mesaros, Leila } & 343 \\ \text { Best, Heinrich } & 156 & \text { Meuleman, Bart } & 263^{*} \\ \text { Best, Henning } & 135^{*} & \text { Moldenhauer, Stephanie } & 491 \\ \text { Blasius, Jörg } & 151 & \text { Mühlau, Peter } & 349 * \\ \text { Bode, Ingo } & 371 & \text { Müller, Albert } & 629 \\ \text { Boudon, Raymond } \dagger & 17 * & \text { Müller, Nils } & 318 \\ \text { Butterwegge, Christoph } & 323 & \text { Müller, Ursula } & 688 \\ \text { Cornelißen, Waltraud } & 488 & \text { Müller-Benedict, Volker } & 115,482 \\ \text { Davidov, Eldad } & 263 * & \text { Nieto Morales, Fernando } & 349 * \\ \text { Deutschmann, Christoph } & 146 & \text { Nollmann, Gerd } & 603 \\ \text { Diefenbach, Heike } & 683 & \text { Nonnenmacher, Alexandra } & 369 *, 1 * \\ \text { Diekmann, Andreas } & 47^{*} & \text { Opp, Karl-Dieter } & 155^{*} \\ \text { Diewald, Martin } & 243 & \text { Pant, Hans Anand } & 165 \\ \text { Dörre, Klaus } & 314 & \text { Papilloud, Christian } & 659 \\ \text { Dütsch, Matthias } & 517 & \text { Pfau-Effinger, Birgit } & 140\end{array}$

1 *Sternchen hinter den Seitenzahlen kennzeichnen Beiträge im 54. Sonderheft, ,Soziale Kontexte und soziale Mechanismen“, hrsg. von Jürgen Friedrichs und Alexandra Nonnenmacher (Wiesbaden: Springer VS).

Die Online-Version des Originalartikels ist unter doi:10.1007/s11577-015-0303-z zu finden. 


\begin{tabular}{|c|c|c|c|}
\hline Egger de Campo, Marianne & 149 & Pöge, Kathleen & 686 \\
\hline Eid, Michael & $189^{*}$ & Pötschke, Manuela & $219^{*}$ \\
\hline Engelage, Sonja & 143 & Preyer, Gerhard & 477 \\
\hline Faulbaum, Frank & 305 & Rausch, Alexander & 77 \\
\hline Fleck, Christian & 629 & Reuband, Karl-Heinz & 306 \\
\hline Friedrichs, Jürgen & $497,1 *, 287^{*}$ & Salheiser, Axel & 507 \\
\hline Frodermann, Corinna & 21 & Schacht, Diana & 445 \\
\hline Galster, George C. & $117^{*}$ & Schanne, Sita & 503 \\
\hline Gebauer, Ronald & 507 & Scharenberg, Katja & $317 *$ \\
\hline Giesselmann, Marco & 95 & Schiek, Daniela & 459 \\
\hline Gückelhorn, Cathrin & 517 & Schimank, Uwe & 657 \\
\hline Hadjar, Andreas & 51 & Schmidt, Peter & $263^{*}$ \\
\hline Hagenah, Jörg & 325 & Schmidt, Rudi & 674 \\
\hline Hainz, Michael & 320 & Schnabel, Anette & 486 \\
\hline Häußling, Roger. & 671 & Schneck, Andreas & 549 \\
\hline Haupt, Andreas & 603 & Schröder, Stefan & 311 \\
\hline Hedmann, Lina & $67 *$ & Schroer, Markus & 193 \\
\hline Heintz, Bettina & 291 & Schwartz, Shalom H. & $263 *$ \\
\hline Heinze, Thomas & 500 & Seyfert, Robert & 666 \\
\hline Himmel, Wolfgang & 328,693 & Shamon, Hawal & 397 \\
\hline Hinz, Thomas & 21,549 & Spier, Tim & $369^{*}$ \\
\hline Holzinger, Markus & 267 & Spitzenpfeil, Martin & 575 \\
\hline Hosoya, Georg & $189^{*}$ & Stanat, Petra & 165 \\
\hline Imbusch, Peter & 491 & Stadtfeld, Christoph & $395^{*}$ \\
\hline Jaehrling, Karen & 343 & Stegbauer, Christian & 77 \\
\hline Kaiser, Till & 243 & Steinbach, Anja & $417 *$ \\
\hline Kalina, Thorsten & 343 & Stephan, Gesine & 517 \\
\hline Kalter, Frank & $91 *$ & Struck, Olaf & 517 \\
\hline Kocaj, Aleksander & 165 & Thieme, Frank & 153,663 \\
\hline Koch, Tobias & $189^{*}$ & Tucci, Ingrid & 445 \\
\hline Köhle-Hezinger, Christel & 669 & Tuppat, Julia & 219 \\
\hline Köhler, Christoph & 311 & Twellmann, Markus & 135 \\
\hline Köthemann, Dennis & 51 & Ullrich, Carsten G. & 459 \\
\hline Krempkow, René & 688 & van Tubergen, Frank & $241 *$ \\
\hline Kristen, Cornelia & 445 & Werron, Tobias & 291 \\
\hline Kroneberg, Clemens & $643,91 *$ & Wilke, Felix & 371 \\
\hline Kroth, Anna J. & 165 & Windzio, Michael & 95 \\
\hline Kuhl, Poldi & 165 & Winter, Rainer & 331 \\
\hline Lepsius, M. Rainer & 159 & Wittek, Rafel & $349 *$ \\
\hline
\end{tabular}




\section{INHALTÜBERSICHT DES 66. JAHRGANGS 2014}

\section{ABHANDLUNGEN}

Andreß, Hans-Jürgen, und Martin Spitzenpfeil: Ist der Anstieg der westdeutschen Einkommensungleichheit auf die Zunahme bildungshomogener Partnerschaften zurückführbar? Eine Dekompositionsanalyse auf Basis des SOEP (1985-2011)

Akyel, Dominic, und Jens Beckert: Pietät und Profit. Kultureller Wandel und Marktentstehung am Beispiel des Bestattungsmarktes

Auspurg, Katrin, Corinna Froderman und Thomas Hinz: Berufliche Umzugsentscheidungen in Partnerschaften. Eine experimentelle Prüfung von Verhandlungstheorie, Frame-Selektion und Low-Cost-These .............

Auspurg, Katrin, Thomas Hinz und Andreas Schneck: Ausmaß und Risikofaktoren des Publication Bias in der deutschen Soziologie

Becker, Oliver Arránz, Daniel Lois und Anja Steinbach: Kontexteffekte in Familien - Angleichung von Paaren und intergenerationale Transmission am Beispiel Religiosität

Best, Henning, und Jochen Mayerl: Theoretische und statistische Modellierung von Cross-Pressures in Kontextanalysen

Bode, Ingo, und Felix Wilke: Orientierungsprozesse im Vertrauensdilemma. Beziehungskonstruktionen in Beratungen zur privaten Altersvorsorge ..... Boudon, Raymond $\dagger$ : What is context?

Davidov, Eldad, Bart Meuleman, Shalom H. Schwartz und Peter Schmidt: Individual values, cultural embeddedness and anti-immigration sentiments: Explaining differences in the effect of values on attitudes towards immigration across Europe

Diekmann, Andreas: Die Anderen als sozialer Kontext. Zur Bedeutung strategischer Interaktion

Dütsch, Matthias, Cathrin Gückelhorn, Gesine Stephan und Olaf Struck: Hohe Gehaltssteigerungen durchs Berufsgewerkschaften: Gerechtigkeitsbewertungen und Folgewirkungen

Friedrichs, Jürgen: Kontexteffekte von Wohngebieten

Friedrichs, Jürgen, und Alexandra Nonnenmacher: Die Analyse sozialer Kontexte

Galster, George C.: Nonlinear and threshold aspects of neighborhood effects

Hadjar, Andreas, und Dennis Köthemann: Klassenspezifische Wahlabstinenz - Spielt das Vertrauen in politische Institutionen eine Rolle?

Haupt, Andreas, und Gerd Nollmann: Warum werden immer mehr Haushalte von Armut gefährdet? Zur Erklärung erhöhter Armutsrisikoquoten mit unbedingten Quantilregressionen

Hedman, Lina: Compositional or contextual effects? Neighbourhoods and teenage parenthood on Stockholm, Sweden 
Hosoya, Georg, Tobias Koch und Michael Eid: Längsschnittdaten und Mehrebenenanalyse

Jaehrling, Karen, Thorsten Kalina und Leila Mesaros: Mehr Arbeit, mehr Armut? Ausmaß und Hintergründe der Entkoppelung von Erwerbsarbeit und materieller Sicherheit von Alleinerziehenden im Ländervergleich ......

Kaiser, Till, und Martin Diewald: Ordentliche Arbeiterkinder, konzentrierte Mittelschichtkinder? Die ungleiche Entwicklung von Gewissenhaftigkeit im frühen Kindesalter

Kalter, Frank, und Clemens Kroneberg: Between mechanism talk and mechanism cult: New emphases in explanatory sociology and empirical research

Kocaj, Aleksander, Poldi Kuhl, Anna J. Kroth, Hans Anand Pant und Petra Stanat: Wo lernen Kinder mit sonderpädagogischem Förderbedarf besser? Ein Vergleich schulischer Kompetenzen zwischen Regel- und Förderschulen in der Primarstufe

Lomi, Alessandro, und Christoph Stadtfeld: Social networks and social settings: Developing a coevolutionary view

Mayntz, Renate: Die Finanzmarktkrise im Licht einer Theorie funktioneller Differenzierung

Nonnenmacher, Alexandra, und Tim Spier: Der Einfluss der Gruppengröße auf die Aktivität von Parteimitgliedern

Opp, Karl-Dieter: Das Aggregationsproblem bei Mikro-Makro-Erklärungen

Pötschke, Manuela: Aktuelle Probleme der Modellierung von MehrebenenDaten

Scharenberg, Katja: Schule und Schulklasse als soziale Kontexte der Entwicklung im Jugendalter

Schroer, Markus: Soziologie der Aufmerksamkeit. Grundlegende Überlegungen zu einem Theorieprogramm

Shamon, Hawal: Ist mein Einkommen gerecht? Die Relevanz von Durchschnittseinkommen, Einkommensverteilung und sozialer Position für Gerechtigkeitsbewertungen

Stegbauer, Christian, und Alexander Rausch: Soziale Beeinflussung in Mikronetzwerken am Beispiel der Erhebung von Markenpräferenzen mit dem „Tischmodell“

Tubergen, Frank van, und Tessel Metjox: Minority language proficiency of adolescent immigrant children in England, Germany, the Netherlands, and Sweden

Tuppat, Julia, und Birgit Becker: Sind türkischstämmige Kinder beim Schulstart im Nachteil? Die Bedeutung genereller und aufnahmelandspezifischer Kompetenzen für die Wahrscheinlichkeit einer Rückstellung ...........

Wittek, Rafael, Fernando Nieto Morales und Peter Mühlau: Evil tidings: Are reorganisations more successful if employees are informed early? 


\section{BERICHTE UND DISKUSSIONEN}

Fleck, Christian, und Albert Müller: Kann das Modell der Frame-Selektion etwas zur „Rettung von Juden im Zweiten Weltkrieg“ beitragen? Eine Kritik an Clemens Kronebergs gleichnamigem Beitrag in der Kölner Zeitschrift für Soziologie und Sozialpsychologie

Giesselmann, Marco, und Michael Windzio: Paneldaten in der Soziologie: Fixed Effects Paradigma und empirische Praxis in Panelregression und Ereignisanalyse

Heintz, Bettina, und Tobias Werron: Fehlinterpretationen der Weltgesellschaftstheorie. Eine Antwort auf Markus Holzingers Kritik der Weltgesellschaftsforschung

Holzinger, Markus: Fehlschlüsse über die „Weltgesellschaft“. Einige Überlegungen im Anschluss an Bettina Heintz' und Tobias Werrons Soziologie des Vergleichs

Kroneberg, Clemens: Understanding Merton. Eine Erwiderung auf Christian Fleck und Albert Müller

Müller-Benedcit, Volker: Grenzen der Meritokratie oder warum Quotenregulierungen sinnvoll sind

Schacht, Diana, Cornelia Kristen und Ingrid Tucci: Interethnische Freundschaften in Deutschland

Ullrich, Carsten G., und Daniela Schiek: Gruppendiskussionen in Internetforen. Zur Methodologie eines neuen qualitativen Erhebungsinstruments

\section{LITERATURBESPRECHUNGEN}

\section{Sammelbesprechung}

Böhle, Fritz, G. Günter Voß und Günther Wachtler (Hrsg.): Handbuch Arbeitssoziologie. Hirsch-Kreinsen, Hartmut, und Heiner Minssen (Hrsg.): Lexikon der Arbeits- und Industriesoziologie (Rudi Schmidt) .....

\section{Liste der rezensierten Bücher}

Andreß, Hans-Jürgen, Katrin Golsch und Alexander W. Schmidt: Applied Panel Data Analysis for Economic and Social Surveys (Frank Faulbaum)

Bauer, Ullrich, Uwe H. Bittlingmayer, Carsten Keller und Franz Schultheis (Hrsg.): Bourdieu und die Frankfurter Schule. Kritische Gesellschaftstheorie im Zeitalter des Neoliberalismus (Christian Papilloud) ... Becker, Jörg: Elisabeth Noelle-Neumann. Demoskopin zwischen NS-Ideologie und Konservatismus (Karl-Heinz Reuand)

Bernt, Matthias, und Heike Liebmann (Hrsg.): Peripherisierung, Stigmatisierung, Abhängigkeit? Deutsche Mittelstädte und ihr Umgang mit Peripherisierungsprozessen (Marcus Menzl) 
Blasius, Jörg, und Victor Thiessen: Assessing the Quality of Survey Data (Volker Müller-Benedict)

Breuer, Marc: Religiöser Wandel als Säkularisierungsfolge. Differenzierungs- und Individualisierungsdiskurse im Katholizismus (Michael Hainz)

Bridge, Gary, Tim Butler und Loretta Lees (Hrsg.): Mixed Communities. Gentrification by stealth? (Jürgen Friedrichs)

Buck, Elena, Anne Dölemeyer, Paul Erxleben, Stefan Kausch, Anne Mehrer, Mathias Rodatz, Frank Schubert, Gregor Wiedemann und Forum für kritische Rechtsextremismusforschung (Hrsg.): Ordnung. Macht. Extremismus. Effekte und Alternativen des Extremismus-Modells (Christoph Butterwegge)

Butterwegge, Christoph, Gerd Bosbach und Matthias W. Birkwald (Hrsg.): Armut im Alter. Probleme und Persepektiven der sozialen Sicherung (Jörg Blasius)

Castel, Robert: Die Krise der Arbeit. Neue Unsicherheiten und die Zukunft des Individuums (Klaus Dörre)

Connell, Raewyn: Confronting Equality: Gender, Knowledge and Global Change (Birgit Pfau-Effinger)

Corsten, Michael, und Michael Kauppert (Hrsg.): Der Mensch - nach Rücksprache mit der Soziologie (Robert Seyfert)

Döge, Peter: Männer - die ewigen Gewalttäter? Gewalt von und gegen Männer in Deutschland (Peter Imbusch und Stephanie Moldenhauer) ..

Dolata, Ulrich, und Jan-Felix Schrape (Hrsg.): Internet, Mobile Devices und die Transformation der Medien. Radikaler Wandel als schrittweise Rekonfiguration (Jörg Hagenah)

Faulbaum, Frank, Matthias Stahl und Erich Wiegand (Hrsg.): Qualitätssicherung in der Umfrageforschung: Neue Herausforderungen für die Markt- und Sozialforschung (Klaus Allerbeck)

Gildemeister, Regine, und Katja Hericks: Geschlechtersoziologie. Theoretische Zugänge zu einer vertrackten Kategorie des Sozialen (Annette Schnabel)

Haller, Max: Rezension von „Best, Heinrich, György Lengyel und Luca Verzichelli (Hrsg.): The Europe of Elites. A Study into the Europeanness of Europe's Political and Economic Elites“ in KZfSS 65 (2013) (Heinrich Best)

Helbig, Marcel: Sind Mädchen besser? Der Wandel geschlechtsspezifischen Bildungserfolgs in Deutschland (Heike Diefenbach)

Klammer, Ute, Sabine Neukirch und Dagmar Weßler-Poßberg: Wenn Mama das Geld verdient. Familienernährerinnen zwischen Prekariat und neuen Rollenbildern (Waltraud Cornelißen)

König, Tomke: Familie heißt Arbeit teilen. Transformationen der symbolischen Geschlechterordnung. Analyse und Forschung (Kathleen Pöge) ...

Kraemer, Klaus, und Sebastian Nessel (Hrsg.): Entfesselte Finanzmärkte. Soziologische Analysen des modernen Kapitalismus (Christoph Deutschmann) 
Lohr, Karin, Thorsten Peetz und Romy Hilbrich: Bildungsarbeit im Umbruch. Zur Ökonomisierung von Arbeit und Organisation in Schulen, Universitäten und in der Weiterbildung (Sonja Engelage)

Mau, Steffen, Heike Brabandt, Lena Laube und Christof Roos: Liberal States and the Freedom of Movement: Selective Borders, Unequal Mobility (Nils Müller)

Münch, Richard: Das Regime des Freihandels. Entwicklung und Ungleichheit in der Weltgesellschaft (Gerhard Preyer)

Niekrenz, Yvonne: Rauschhafte Vergemeinschaftungen. Eine Studie zum rheinischen Straßenkarneval. Erlebniswelten (Christel Köhle-Hezinger)

Peter, Claudia, und Dorett Funcke (Hrsg.): Wissen an der Grenze: Zum Umgang mit Ungewissheit und Unsicherheit in der modernen Medizin (Markus Twellmann)

Promberger, Markus: Topografie der Leiharbeit - Flexibilität und Prekarität einer atypischen Beschäftigungsform (Christoph Köhler und Stefan Schröder)

Reinhart, Martin: Soziologie und Epistemologie des Peer Review (Thomas Heinze)

Rosenberger, Sieglinde, und Birgit Sauer (Hrsg.): Politics, Religion und Gender. Framing and regulating the veil (Marianne Egger de Campo) ..

Schneickert, Christian: Studentische Hilfskräfte und MitarbeiterInnen. Soziale Herkunft, Geschlecht und Strategien im Wissenschaftlichen Feld (Sita Schanne)

Schnitzler, Sonja: Soziologie im Nationalsozialismus zwischen Wissenschaft und Politik. Elisabeth Pfeil und das „Archiv für Bevölkerungswissenschaft und Bevölkerungspolitik“" (Frank Thieme)

Schönhuth, Michael, Markus Gamper, Michael Kronenwett und Martin Stark (Hrsg.): Visuelle Netzwerkforschung. Qualitative, quantitative und partizipative Zugänge (Roger Häußling)

Schwinn, Thomas: Max Weber und die Systemtheorie (Uwe Schimank) ........

Sofsky, Wolfgang: Einzelgänger (Wolfgang Himmel)

Vogel, Claudia, und Andreas Motel-Klingebiel (Hrsg.): Altern im sozialen Wandel: Die Rückkehr der Altersarmut? (Frank Thieme)

Wilkesmann, Uwe, und Christian J. Schmid (Hrsg.): Hochschule als Organisation (René Krempkow und Ursula Müller)

\section{NACHRICHTEN UND MITTEILUNGEN}

\section{Nekrolog}

Lepsius, M. Rainer: Nachruf auf Juan J. Linz (24. Dezember 1926-1. Oktober 2013)

Schluchter, Wolfgang: Nachruf auf M. Rainer Lepsius (18. Mai 1928-2.

Oktober 2014) 
Winter, Rainer: Nachruf auf Stuart Hall (3. Februar 1932-10. Februar 2014)

\section{Würdigung}

Gebauer, Ronald, und Axel Salheiser: Heinrich Best zum 65. Geburtstag ...

\section{Mitteilungen}

Wolfgang Kaupen-Preis 2013 für Gunther Teubner

Schader-Preis 2014 für Stephan Leibfried

Berliner Wissenschaftspreis an Bildungssoziologin Heike Solga verliehen ...

Preis der Fritz Thyssen Stiftung für sozialwissenschaftliche Aufsätze (Begründet durch Prof. Dr. Dr. h.c. Erwin K. Scheuch $\dagger$ ), Zeitschriftenjahrgang 2013

\section{Ankündigungen}

Gemeinsamer Kongress der Deutschen Gesellschaft für Medizinische Psychologie und der Deutschen Gesellschaft für Medizinische Soziologie, 14.-20. September 2014, Greifswald

„Routinen der Krise - Krise der Routinen”, Themenpapier zum 37. Kongress der Deutschen Gesellschaft für Soziologie, 6.-10. Oktober 2014, Trier

Nutzerkonferenz „Forschen mit dem Mikrozensus - Analysen zur Sozialstruktur und zum sozialen Wandel“, 11.-12. November 2014, Mannheim

Tagung „Die DDR im sozialen Gedächtnis - Theoretische und empirische Zugänge“, 12.-13. März 2015, Wissenschaftszentrum Berlin (WZB) .......

Tagung „Die diskursive Konstruktion von Wirklichkeit II. Interdisziplinäre Perspektiven einer wissenssoziologischen Diskursforschung“", Universität Augsburg, 26.-27.März 2015

Tagung „Das bedrohte Selbst. Formen der Subjektivierung zwischen Kontrolle und Eigen-Sinn 1915-2015." Wissenschaftliche Tagung der Promovierenden ....2015, Georg-August-Universitat Göttingen - Historische Sternwarte, 21.-23. April 2015

\section{Call for Papers}

„feministische studien“, Call for Papers für Heft 2/2014, Themenschwerpunkt: "Sex in the City: Frauen im öffentlichen Raum"

Nutzerkonferenz „Forschen mit dem Mikrozensus“, Analysen zur Sozialstruktur und zum sozialen Wandel, 11.-12. November 2014, Mannheim ..

Freiburger Zeitschrift für Geschlechter Studien (fzg) zum Thema: „Medizin - Gesundheit - Geschlecht“

Qualitative Online-Erhebungen, Tagung an der Universität Duisburg-Essen, 16.-17. Januar 2015 
Microdata from Eurostat, SILC, LFS, AES, SES, CIS, EHIS and HBS, 4th European User Conference, March 5-6, 2015, Mannheim

Call for papers für ein Sonderheft der Österreichischen Zeitschrift für Soziologie: „Handlungs- und Interaktionskrisen - Theoretische und empirische mikro-soziologische Perspektiven“, geplante Veröffentlichung: Frühjahr 2016

Social Norms and Institutions. Model Building, Empirical Corroborations, and Applications. International Conference at the Congressi Stefano Franscini (CSF) of ETH Zurich, Monte Verità, Ascone 10-15

May 2015

\section{Redaktionelles}

Erratum

693

Editorial

Gutachterinnen und Gutachter der Kölner Zeitschrift für Soziologie und Sozialpsychologie 2013/2014 